\title{
A DATA ACQUISITION SYSTEM WITH ON-LINE COMPUTER
}

\section{ERIK HøG}

Hamburger Sternwarte, Hamburg, D.B.R.

Nather and Evans (1970) emphasize that merely recording the light curve of an occultation is not enough. The data must be recorded in a form which is convenient for the analysis to be carried out later on. Analog methods of recording merely delay the time at which the record must be measured, usually after it has been digitized in some way. Nather and Evans have used a modified digital multiscaler with 400 storage locations for the observation. The data acquisition to be described here is in principle rather similar to a multiscaler and deserves special attention because it is in practice even more flexible and convenient due to its on-line computer. Our system has been used to measure star occultations (De Vegt and Pansch, 1970) and the Crab pulsar (Høg and Lohsen, 1970) and to sample data from a scanning photometer (Høg, 1969).

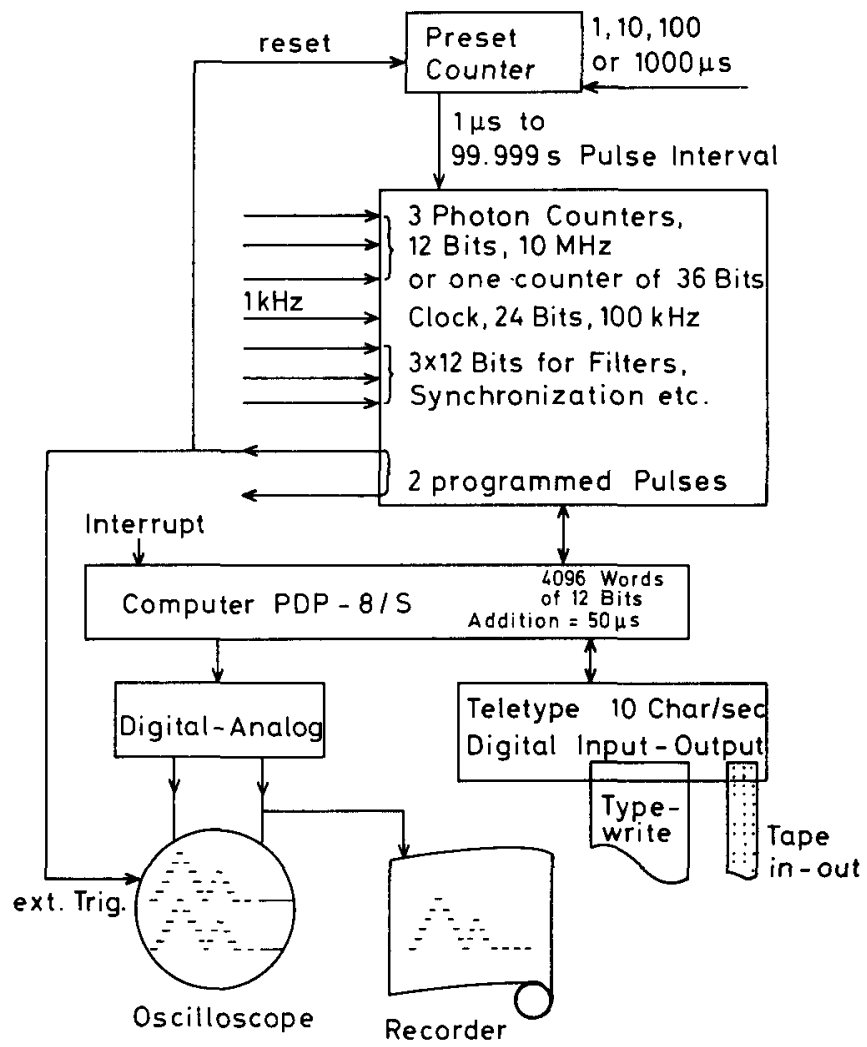

Fig. 1. The data acquisition system with on-line computer. It may be used for sampling up to 3000 measurements per second at accurately timed intervals. The computer may request 8 different external buffers of 12 bits each connected with counters etc. It has analog and digital outputs. 
The main item of our system, Figure 1, is a PDP-8/S computer with a digital inputoutput on a teletype (10 characters/sec). Two digital-to-analog converters provide output on an oscilloscope and on a strip chart recorder giving the observer an opportunity to check immediately the validity of the results and to produce illustrative documentation from the observations.

Input of data to the computer occurs on request from the computer program which may address 8 words of 12 bits each. Furthermore the computer may emit two short pulses, one of which is used to trigger the oscilloscope. The 8 words are distributed as shown in Figure 1: 3 buffers of photon counters, 1 buffer of a 24 bit clock counter and 3 words for digitizing filter wheels etc. The photon counters are used for direct pulse counting of the photoelectrons. They are stopped within $1 \mathrm{~m}$.sec by the timing pulse from the preset counter in order to transfer the content of the counters to their respective buffers, and reset the counters. While the photons lost during this short stop of the counter are negligible, it is not permissible to stop the clock counter during the transfer. Therefore the clock counter is open to count clock pulses all the time and transfers its content to its buffer after each clock pulse, except when this transfer has been inhibited by a computer instruction. Other instructions provide for transfer of the content of any of the counter buffers to the accumulator of the computer, for resetting the clock counter and for re-permitting the transfer from clock counter to clock buffer. The timing signal from the preset counter sets a status flip-flop which may be requested by the computer waiting in a loop for this status to be set. When the status flip-flop has been set, the program must transfer the contents of the buffers to the storage of the computer and reset the status flip-flop in due time before the next timing signal from the preset counter arrives.

The counters and interface were built in our workshop with integrated circuits (TTL-logic).

The clock counter may be synchronized with UT to within $\pm 1 \mathrm{msec}$ in the following way. A $1 s$-pulse from the external UT clock is connected to one of the 36 bit-lines. At the very beginning of the program a loop is set up which requests this bit until the $1 s$-pulse occurs. After the loop the program will reset the clock counter to zero which will correspond to the second of UT manually noted by the operator when starting the program.

This system has cost $\$ 15000$ plus two man-years and has been in operation since June 1969. Similar systems will be in operation on many telescopes in the near future so that stellar occultations can be measured in between other photometric observations once the software system is developed.

\section{References}

Høg, E.: 1969, Proceedings of the IAU Colloquium No. 5 on Visual Double Stars, Nice 1969.

Høg, E. and Lohsen, E.: 1970, Nature 227, 1229.

Nather, R. E. and Evans, D. S.: 1969, 'Discovery and Measurement of Double Stars by Lunar Occultations', Astron J. (preprint).

de Vegt, Chr. and Pansch, E.: 1970 in Joint Discussion on Photoelectric Observation of Stellar Occultations. 